\title{
Optimal UAV Base Station Trajectories Using Flow-Level Models for Reinforcement Learning
}

\author{
Vidit Saxena, Student Member, IEEE, Joakim Jaldén, Senior Member, IEEE, and Henrik Klessig
}

\begin{abstract}
Cellular base stations (BS) and remote radio heads can be mounted on unmanned aerial vehicles (UAV) for flexible, traffic-aware deployment. These UAV base station networks (UAVBSN) promise an unprecendented degree of freedom that can be exploited for spectral efficiency gains as well as optimal network utilization. However, the current literature lacks realistic radio and traffic models for UAVBSN deployment planning and for performance evaluation. In this paper, we propose flowlevel models (FLM) for realistically characterizing the UAVBSN performance in terms of a broad range of flow- and systemlevel metrics. Further, we propose a deep reinforcement learning (DRL) approach that relies on the UAVBSN FLM for learning the optimal traffic-aware UAV trajectories. For a given user traffic density and starting UAV locations, our RL approach learns the optimal UAV trajectories offline that maximizes a cumulative performance metric. We then execute the learned UAV trajectories in a discrete event simulator to evaluate online UAVBSN performance. For $M=9$ UAVs deployed in a simulated Downtown San Francisco model, where the UAV trajectories are defined by $N=20$ discrete actions, our approach achieves approximately a three-fold increase in the average user throughput compared to the initial UAV placement, while simultaneously balancing traffic loads across the BSs.
\end{abstract}

Index Terms-UAV Base Stations, Flow-level models, Reinforcement learning, Proximal Policy Optimization.

\section{INTRODUCTION}

Due to recent advances in unmanned aerial vehicle (UAV) technology and battery lifetime, novel drone-based applications emerge [1]. UAVs are also considered in the wireless communications domain and are treated from two perspectives: UAVs as user equipment (UE) and UAVs as mobile base stations (BSs) providing wireless connectivity to other aerial nodes or ground UEs. While UAVs as UEs have actively been studied for quite some time and study results already found their way into standardization [2], UAVs as BSs are being considered as a deployment option for beyond-5G or $6 \mathrm{G}$ networks [3] only recently. Here, mobile UAV BSs can either be used to build up a wireless access network adhoc for disaster relief or to complement terrestrial, cellular BS deployments that feature fixed, tower-mounted radios and corresponding immobile baseband units.

In general, UAV BSs are classified according their hovering height. While high altitude platforms [4] that hover

Vidit Saxena is with the Department of Information Science and Engineering, KTH Royal Institute of Technology, Stockholm, and with Ericsson Research, Stockholm. Dr. Joakim Jaldén is with the Department of Information Science and Engineering, KTH Royal Institute of Technology, Stockholm. Dr Henrik Klessig is with International Computer Science Institute, Berkeley, CA, and with University of California, Berkeley, CA.

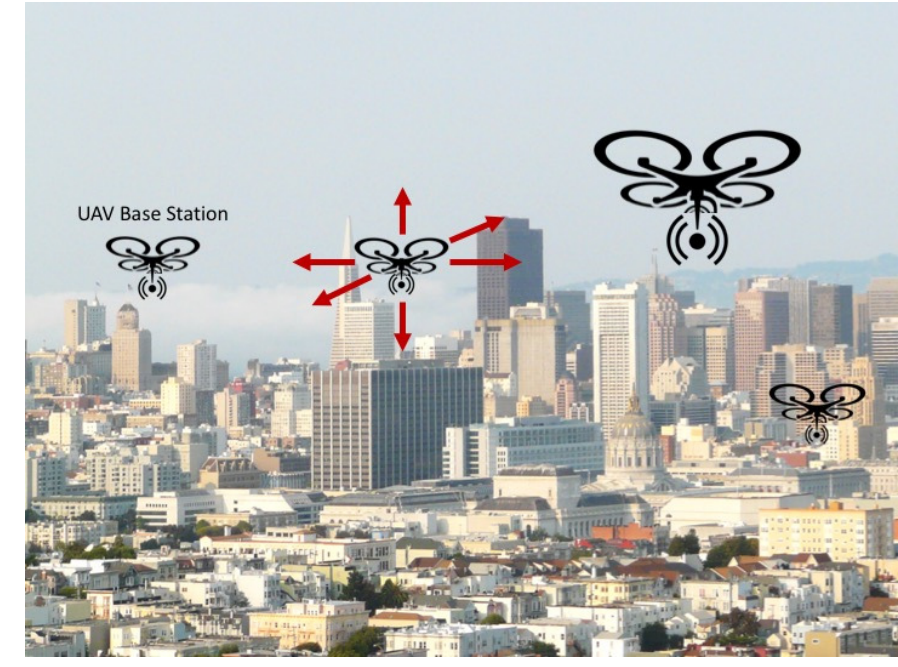

Fig. 1. Mockup of UAV Base Stations (BS) deployed over downtown San Francisco. The red arrows denote the six possible directions for UAV motion along the three orthogonal spatial axes. In this paper, we propose a deep reinforcement learning (DRL) approach for predicting optimal UAV BS trajectories, which maximizes spectral efficiency while also balancing the traffic loads across the BSs. (CKitchen from San Francisco, Julian Herzog [CC BY 4.0]).

at extremely high altitudes of several kilometers, such as helium balloons [5], have been studied for several years, cellular access points mounted onto low-altitude platforms (LAPs) are gaining interest just recently [6]-[9]. UAV BS networks, flexibly deployed between $100 \mathrm{~m}$ to $10,000 \mathrm{~m}$ above the ground, can provide wireless capacity in an extremely adaptive, on-demand fashion to mobile users [9], [10]. In addition, aerial distributed antenna systems (ADASs) [11][13] can be a complementary technology to further improve data rates at traffic hot spots, which would otherwise cause congestion. Furthermore, cloudlets, that are compact microdatacenters, mounted onto some of the UAV BSs can provide on-demand, low-latency edge computing power at construction sites, hazardous locations or for consumers [14], [15]. Such massive aerial networks are likely to cope with the increasing data rate requirements in the Internet of Things era.

While ultra-dense cellular, terrestrial networks composed of small cells have so far been considered the best solution to capacity bottlenecks originating from the ever-increasing traffic explosion, they still lack flexibility in serving spatially varying traffic demand efficiently because of their stationary positions. In particular, during high-traffic times, some small 
cells can be overloaded leading to very low individual user throughputs and high inter-cell interference. Then, interference mitigation techniques are mostly limited. Complementary or alternatively to small cells, UAV BS networks (UAVBSN) seem to be an attractive solution to time- and spatially-varying traffic demands, where network utilization is often either very low, when too many BSs are serving too few users, or extremely high, when there are far more users than planned at the time of deployment. UAV BSs can dynamically follow the traffic hotspots over time, while providing on-demand wireless capacity with optimal radio resource utilization, which is both, energy-efficient and beneficial for the served UEs in terms of per-UE throughput. When traffic loads are balanced among the UAV BSs, interference mitigation techniques have a higher potential to further improve the system's spectral efficiency.

Nevertheless, predicting optimal trajectories for a network of UAV BSs is anything but trivial due to the very high degree of freedom and the variety of different traffic realizations. In Fig. 1, we illustrate this problem through the mockup of a UAVBSN deployed over an urban area. Each UAV BS within the network can be moved along the three orthogonal spatial axes in response to the ambient traffic conditions in the served area. The trajectory optimization problem for UAV BSs can also be viewed as a high-dimensional path planning problem, where the goal is to maximize some cumulative reward metric within a stochastic traffic environment. Recently, deep reinforcement learning (DRL), which learns from random exploration of the environment that they operate in, has achieved breakthrough success in optimizing complex online tasks. DRL now outperforms humans in several problems that involve high-dimensional observed states and planning over several timesteps, e.g., at playing video games [16], beating human experts at Go [17], and controlling robots [18].

Motivated by the complexity of the UAV trajectory planning problem, in this article, we use DRL in combination with flowlevel models (FLMs) so solve the complex problem of finding optimal UAV trajectories for improved spectral efficiency and, at the same time, for more balanced radio utilizations across the UAV BSs. We use FLMs as a realistic model for the network performance in response to traffic variations to help the DRL learn the relationships between the environment and key performance indicators. We show that this approach achieves a considerable improvement of the average data flow throughput in comparison to the initial deployment that is determined according to circle packing theory.

\section{A. Organization of the Article}

Some related work along with their limitations are presented in Section II. We also highlight, which of the limitations are overcome by our approach. We introduce the general system model of the wireless system and the service of data flows in Section III and provide our FLM-based DRL approach in Section IV. In SectionV, we provide details regarding the implementation of our system, and describe the evaluation scenario and numerical results. Section $\mathrm{VI}$ concludes the article.

\section{RELATED WORK}

The deployment and optimization of UAVBSNs has attracted significant research interest recently owing to their flexibility and performance potential. Several approaches have been developed for optimal deployment of UAV BSs over a serving area, for example in [19] and the references therein, and the reinforcement learning approach proposed in [20]. However in this paper, we are interested in the more complex online problem of trajectory optimization, where the deployed UAV BSs are moved to dynamically improve the network performance. In [21], a theoretical model for the energy consumption of fixed-wing UAVs was developed, which was exploited to design an energy-efficiency UAV trajectory. For non-fixed wing UAVs that provide significantly more movement degrees of freedom, trajectory optimization with communication constraints were investigated in [22] and [23]. Further in [24], UAV BS trajectories were optimized with a goal to offload data from traditional, fixed, BSs to improve the overall network performance. In [25], functional programming and optimal control techniques were investigated to find optimal trajectories in terms of maximizing the average sum rate for terminals over the trajectory. This approach was extended in [26] to account for limited flying times for the UAVs by incorporating pre-defined landing spots. Finally in [27], a reinforcement learning approach was developed for two-dimensional UAV BS trajectory optimization where a neural-network model was demonstrated to outperform table-based learning techniques.

\section{A. Limitations of Existing Approaches}

Despite the increasing interest in UAVBSN networks, a systematic, comprehensive and coherent approach to model and dynamically re-arrange large clusters of UAV BSs is nonexisting. This is mainly due to two problems: Firstly, an accurate yet tractable system model to assess such networks at a large scale and in a holistic manner is missing. Existing models rely on simple system assumptions, for example, omnidirectional antennas [28]-[30], [30]-[41] and average line-ofsight (LOS)/non-line-of-sight (NLOS) conditions [29]-[31], [36]-[44], and consider one single [30]-[32], [42], [43], [45] or only a handful of UAV BSs [28], [29]. Also, inter-cell interference, which is harder to coordinate in mobile UAV BS networks [6], is often assumed to be full interference [28], [30], [32]-[35], [44]-[47], resembling an extreme case. Furthermore, models fail to integrate realistically the power consumption of UAVs and BS equipment. Secondly, threedimensional placement of a limited number of UAV BSs does not go beyond deployment with coverage constraints [28], [31], [37], [38], [42]-[45]. Large-scale, flexible and dynamic re-arrangement of aerial nodes for capacity provisioning and load balancing in response to spatio-temporal data demand fluctuations while considering backhauling load, network energy efficiency, and computing has not been addressed so far. Very few studies on UAV-cloudlets, such as in [14], address the problem of minimizing the energy consumption of mobile users, but neglect latency, wireless link load and network energy efficiency. 


\section{B. Contributions of this Article}

In this article, we provide an approach that combines DRL with FLM, such that an agent is able to learn optimal UAV BS trajectories for a multitude of aerial nodes. We define optimality in terms of balancing the traffic load across the UAV BSs while simultaneously maximizing the total network throughput. While showing the potential of this approach, we also tackle some of the problems mentioned above. In particular, we make the following contributions:

1) Instead of considering statistical LOS (neither probabilistic LOS nor averaging LOS and NLOS link budgets), our approach considers empirical LOS conditions, which we show by means of a realistic, threedimensional model of Downtown San Francisco.

2) In addition, our FLM-based approach holistically takes into account arbitrary user and traffic distributions, spectral efficiency, and BS radio utilizations as opposed to coverage optimization only in many other works. Optionally, the FLM can also be used to optimize UAV BS trajectories towards increased energy efficiency, system capacity, and other metrics.

3) The algorithm does neither limit the system to omnidirectional antennas nor does it assume idealistic or worst-case interference among BSs as opposed to the state-of-the-art. In contrast, it explicitly considers dynamic inter-cell interference by means of random activity of neighboring UAV BSs based on traffic statistics.

4) Because the DRL agent learns UAV BS trajectories based on the output of a model, i. e., the FLM, learning happens offline and arbitrarily fast depending on the used computing resources. Actual trajectories are then optimized based on empirical flow service statistics (for which the FLM provides a steady-state distribution), which can, in reality, be obtained from scheduling information at each UAV BS.

\section{SYSTEM MODEL}

In this section, we describe the system model, which is the common basis for both, the FLM and the simulator, which we use to evaluate the DRL agent's performance. Simulation- and FLM-specific definitions and derivations are provided in the respective paragraphs in SectionIV.

\section{A. UAV Base Station Network}

We consider a region $\mathcal{L} \subset \mathbb{R}^{3}$ served by $M$ UAV base stations (BSs) indexed by $m \in \mathcal{M}:=\{1, \ldots, M\}$. The UAV BSs are airborne wireless access points serving mobile users located at ground level. In this paper, we only consider downlink user traffic served by the UAVBSN. The UAV BSs can be located within the region $\mathcal{L}^{\prime} \subset \mathcal{L}$, which restricts the maximum UAV elevation, $h_{\mathrm{UAV}}^{\max }$, as well as the minimum UAV elevation above the ground plane, $h_{\mathrm{UAV}}^{\min }$. Further, the spatial locations of the UAV BSs vary over time in response to radio and user traffic conditions. We collect the time-dependent UAV BS locations in the set $\mathcal{U}_{\mathrm{UAV}}(t):=\left\{u_{\mathrm{UAV}}^{(1)}, \ldots, u_{\mathrm{UAV}}^{(M)}\right\}(t)$, where $u_{\mathrm{UAV}}^{(m)} \in \mathcal{L}^{\prime}$.

\section{B. Data Traffic Model}

Our traffic model follows the definition of data flows [48]. A data flow is defined as a number of Internet Protocol (IP) packets that belong to a certain object, such as a webpage or a video file. Data flows arrive randomly in time and space, as well as independent of each other, based upon a temporally inhomogeneous Poisson process and, at time instant $t$, according to a true spatial traffic distribution $\sigma(u, t)$ for all $u \in \mathcal{L}$. Each data flow is of a certain size given in bits, drawn from a probability distribution with mean $\Omega$, and is labeled by its location. The locations of $L$ active data flows at time $t$ are collected in the set $\mathcal{U}_{\mathrm{UE}}(t)=\left\{u_{\mathrm{UE}}^{(1)}, \ldots, u_{\mathrm{UE}}^{(L)}\right\}(t)$. We assume that any UAV BS $m \in \mathcal{M}$ can serve at most $D_{m}$ data flows at any given point in time, so that $L \leq \sum_{m \in \mathcal{M}} D_{m}$. As users are potentially mobile, data flow locations can vary over time $t$.

\section{Service of a Data Flow}

At each time instant $t$, each data flow is uniquely associated with a UAV BS according to some fixed user association rule. The service of a data flow by that BS is characterized by the service time, which is the duration between the flow's arrival and departure. It departs from the system, once the entire amount of information has been transmitted. The service time is determined by the link budget of the communication channel between the user equipment and the access point, the fact that radio resources are dynamically shared among multiple data flows (scheduling), non-deterministic inter-cell interference, and the size of the data flow. The throughput of a data flow is the ratio of its size and its service time, given in bps. At each time $t$, BS $m \in \mathcal{M}$ serves $X_{m}(t) \leq D_{m}$ concurrent data flows that are associated with it. We collect the numbers of data flows for all BSs in the vector $X(t)=\left(X_{1}(t), \ldots, X_{M}(t)\right)^{\top}$. Note that we have $\|X(t)\|_{1}=\left|\mathcal{U}_{\mathrm{UE}}(t)\right|$.

\section{Radio Propagation and Signal Quality}

Each connection of a UAV BS $m$ to a user (or data flow) at location $u_{\mathrm{UE}}^{(l)}(t)$ is characterized by the link budget between the UAV's location $u_{\mathrm{UAV}}^{(m)}(t)$ and the user's location $u_{\mathrm{UE}}^{(l)}(t)$, which has multiple components. In particular, the receive power in $\mathrm{dBm}$ at the UE's location $u_{\mathrm{UE}}^{(l)}(t)$ from UAV BS $m$ is given as

$$
\begin{gathered}
p_{m}^{\mathrm{dBm}}\left(u_{\mathrm{UE}}^{(l)}(t)\right)=p_{m, \mathrm{tx}}-P L\left(u_{\mathrm{UE}}^{(l)}(t), u_{\mathrm{UAV}}^{(m)}(t)\right) \\
+A_{m}\left(u_{\mathrm{UE}}^{(l)}(t), u_{\mathrm{UAV}}^{(m)}(t)\right)+D_{\mathrm{MIMO}}-M_{\mathrm{FF}} \\
-N F_{\mathrm{UE}}-N F_{\mathrm{BS}}-N L\left(u_{\mathrm{UE}}^{(l)}(t), u_{\mathrm{UAV}}^{(m)}(t)\right),
\end{gathered}
$$

where $p_{m, \mathrm{tx}}$ is the maximum transmit power of UAV BS $m$, $A_{m}$ is the antenna gain of UAV BS $m$, which depends on the 
angle between both locations, $D_{\text {MIMO }}$ is an average MIMO gain, $M_{\mathrm{FF}}$ is a fast fading margin, and $N F_{\mathrm{UE}}$ and $N F_{\mathrm{BS}}$ are noise figures at the receiver and transmitter, respectively. The quantity $N L$ is a LOS/NLOS offset, which depends on whether there is LOS between both locations or not as well as on the environment, i. e. the built-up area. It is important to note that, in contrast to existing work, we consider empirical LOS/NLOS conditions that depend on the three-dimensional structure of the environment and the positions of the UAV BSs and the UEs. Also, the antenna gain, $A_{m}$, does not need to be tailored to a specific one; it rather can be any arbitrary antenna model. Without loss of generality, we assume that the path loss $P L$ follows a free-space propagation model given by

$$
\begin{aligned}
P L & \left(u_{\mathrm{UE}}^{(l)}(t), u_{\mathrm{UAV}}^{(m)}(t)\right)= \\
& 20 \log _{10}\left(\frac{4 \pi f_{\mathrm{c}} d\left(u_{\mathrm{UE}}^{(l)}(t), u_{\mathrm{UAV}}^{(m)}(t)\right)}{c}\right),
\end{aligned}
$$

with $f_{\mathrm{c}}$ being the carrier frequency, $d$ a Euclidean distance measure, and $c$ the speed of light. From (1) and (2), it is clear that by exploiting the high spatial degrees of freedom, a UAVBSN can substantially optimize the UE received powers. Although, users can in principle be mobile, in the model, we assume the user speed slow enough that macro-scale (viz. shadowing) receive power conditions remain approximately static for the duration of an active data flow [49]. Mobility is then modeled as redrawing the location of another subsequent data flow by the same user.

We acknowledge that signal quality is affected by inter-cell interference, which is heavily determined by the UAV BS locations and also the temporal traffic patterns within the interferers' cells. We capture this behavior in the description of the SINR

$$
\gamma_{m}\left(u_{\mathrm{UE}}^{(l)}(t), Y(t)\right):=\frac{p_{m}\left(u_{\mathrm{UE}}^{(l)}(t)\right)}{\sum_{n \in \mathcal{M} \backslash\{m\}} Y_{n}(t) p_{n}\left(u_{\mathrm{UE}}^{(l)}(t)\right)+N_{0}},
$$

where the terms $Y_{n}(t):=\operatorname{sgn}\left(X_{n}(t)\right)$ account for the fact that a neighboring BS interfers if it serves at least one active data flow and where $p_{m}$ is the receive power on absolute scale. Accordingly, we define the vector $Y(t):=\left[Y_{1}(t), \ldots, Y_{M}(t)\right]^{\top}$. Utilizing the Shannon capacity, the maximally achievable data rate of a user at location $u_{\mathrm{UE}}^{(l)}(t) \in \mathcal{L}_{m}$ is given as

$$
\begin{aligned}
& c_{m}\left(u_{\mathrm{UE}}^{(l)}(t), Y(t)\right):= \\
& \quad B \min \left\{\log _{2}\left(1+\gamma_{m}\left(u_{\mathrm{UE}}^{(l)}(t), Y(t)\right)\right), c_{\max }\right\} .
\end{aligned}
$$

Here, the quantities $B$ and $c_{\max }$ determine the system bandwidth and the data rate according to the highest modulation and coding scheme available for transmission.

\section{E. Service Dynamics}

We assume that the UAV BSs have schedulers, which allocate radio resources fairly among data flows irrespective of their achievable data rates $c_{m}$. Then, the individual, instantaneous flow throughput is given as

$$
r_{m}\left(u_{\mathrm{UE}}^{(l)}(t), X_{m}(t), Y(t)\right)=\frac{c_{m}\left(u_{\mathrm{UE}}^{(l)}(t), Y(t)\right)}{X_{m}(t)} .
$$

It is important to note that both, $X_{m}(t)$ and $Y(t)$, are subject to dynamic changes during the lifetime of a flow, effectively modulating the flow's throughput according to the underlying data traffic dynamics within the flow's cell as well as within the cells of its neighbors. The time-variant flow throughput along with its flow size determine its lifetime. We assume that the scheduler in each UAV BS tracks the statistics of the number $X_{m}(t)$ of concurrently served flows over time.

\section{UAVBSN TRAJECTORY OPTIMIZATION}

In this section, we first define general requirements on the optimization approach and give some insights into our motivation to use FLM. Then, we formulate the optimization problem for the agent and show how to solve it using DRL.

\section{A. Requirements on the Optimization Approach}

The problem of finding proper UAV BS trajectories is very complex due to the high degree of freedom and due to randomness of the underlying traffic distribution and temporal traffic statistics, viz. random arrivals and departures of data flows. Generally speaking, the online optimization in a real network, hence, must be characterized by a fast, efficient and robust adaptation of the locations of the UAV BSs in accordance to spatio-temporal traffic fluctuations. Fast means that a minimum number of training iterations is required until a near optimal solution is found. Also, the true, spatial traffic distribution changes over time with non-negligible differences in time spans of around one hour, which means that adaptation should happen within a few minutes. Efficient refers to the requirement that the the amount of online exploration of the environment is minimized, since each online exploration incurs the time and energy costs of moving the UAVs in the real environment. The optimization must also be robust, which means that intermediate locations of the UAV trajectories do not deteriorate network performance. In this regard, the agent should not react to individual data flow arrivals or departures, because, on the one hand, randomness in the process can produce atypical flow constellations leading to sub-optimal UAV BS trajectories and, on the other hand, data flow dynamics may happen on much smaller time scales than UAV movements making the latter inefficient.

The FLM from [49] can help fulfill the requirements. In particular, offline learning using the FLM can make the actual online optimization fast and robust. Robustness is also increased by using a-priori information and/or expert knowledge, which is inherent in the FLM. Because the FLM computes an estimate on the steady-state distribution of the number $X_{m}$ of concurrently active data flows served by UAV BS $m$, it fosters efficiency and robustness too. 


\section{B. Problem Formulation for the Learning Agent}

We begin by defining discrete, fixed-duration time intervals, $n \in\{1, \ldots, N\}$. During each time interval, the UAVs hover at their current location and serve the incoming flows. The UAVs additionally estimate the network state state over the time interval and feed this information to a central agent, which predicts the next motion of the UAVs to be executed at the end of the time interval. The UAVs therefore undergo $N$ motions, which constitute the UAVBSN trajectory to be optimized. We denote the set of UAV locations during time interval $n$ with $\mathcal{U}_{\text {UAV }}(n)$, with a slight abuse of the continuous-time notation introduced earlier. Further, to characterize the network state during time interval $n$, each UAV calculates the fraction of time during which it concurrently served $\left\{0, \ldots, D_{m}\right\}$ flows, where $m \in\{1, \ldots, M\}$ is the UAV index. This quantity provides a compact snapshot of the load on each UAV, and is conveniently formulated as the probability distribution over $X_{m}(n)$

$$
\begin{aligned}
\mathcal{P}(n)= & {\left[\operatorname{Pr}\left[X_{1}(n)=0\right], \ldots, \operatorname{Pr}\left[X_{1}(n)=D_{1}\right], \ldots,\right.} \\
& \left.\operatorname{Pr}\left[X_{M}(n)=0\right], \ldots, \operatorname{Pr}\left[X_{M}(n)=D_{M}\right]\right] .
\end{aligned}
$$

We use this distribution to define the observed network state during the time interval $n$,

$$
\mathcal{S}(n):=\left\{\mathcal{U}_{\mathrm{UAV}}(n), \mathcal{P}(n)\right\},
$$

which captures the locations of the UAVs and the load distribution for each UAV. This state is readily available in the existing cellular networks, and does not incur any additional estimation cost at the UAV BS. However, the state for each UAV needs to be communicated to a central node to predict the next UAV motion to be executed. For most traffic scenarios, the UAVs need to be moved infrequently compared to the average flow duration for the served users. Therefore, the additional overhead of communicating the network state is expected to be small as well.

The advantage of UAV BSs is that they can be moved freely through space to optimize the network. However, to learn the optimal trajectories, the agent would need to numerically evaluate arbitrary UAV motion, which would requires an infeasible amount of computational resources. Instead, we decompose the motion of each UAV into the three orthogonal spatial directions. At the beginning of each time interval, the location of each UAV BS is updated through the discrete action scalar $a_{m}(n) \in\{1, \ldots, 7\}, m \in \mathcal{M}$, where $a_{m}(n)=1$ denotes that the UAV location is not changed, and the values $a_{m}(n)=\{2, \ldots, 7\}$ denote UAV movement by a fixed distance in one of six available directions for UAV movement - North, East, South, West, Up, and Down respectively.

The step distance moved by the UAV is denoted by $\Delta u_{h}$ for horizontal actions and by $\Delta u_{v}$ for vertical actions. We collect the UAV actions in $\mathcal{A}(n):=\left\{a_{1}(n), \ldots, a_{m}(n)\right\}$. We assume that the UAV BS locations do not change during any time interval. Instead the UAV BSs move to their new locations

$$
\mathcal{U}_{\mathrm{UAV}}(n+1) \leftarrow\left(\mathcal{U}_{\mathrm{UAV}}(n), \mathcal{A}(n)\right)
$$

in a negligibly small amount of time, and hover at this location until the next action is executed. The UAVs serve traffic during the entire hovering time. At the start of a new time interval, the updated UAV positions are calculated and the UE associations to the BS are updated according to the user association rule. If a user with an active data flow encounters a change in the serving BS, the flow is handed over to the new BS if capacity is available, or retained with the previous BS otherwise.

Since the UAV BS locations are Markovian (i.e., they depend only on the location in the preceding interval and actions takes in that interval), the network state defined in (6) can is a Markov process as well, leading to the following definition of the state transition probability

$$
\operatorname{Pr}[\mathcal{S}(n+1) \mid \mathcal{S}(n), \mathcal{A}(n)],
$$

which depends only on the state and action at time interval $n$.

We are now ready to describe the goal of trajectory optimization. For UAVBSNs, trajectory optimization is the problem of maximizing the trajectory reward, $\sum_{n=1}^{N} \mathcal{R}(n)$, where $\mathcal{R}(n) \in \mathcal{R}$ is some well-defined network performance metric that depends on the observed network state $\mathcal{S}(n)$. Our goal therefore is to find a fast, efficient, and robust policy $\pi_{\theta^{*}}$ for UAV motion that maximizes the trajectory reward, i.e.,

$$
\pi_{\theta^{*}}=\arg \max _{\pi_{\theta}} \sum_{n=1}^{N} \mathcal{R}(n),
$$

where $\theta$ are the trainable parameters for the UAVBSN motion selection policy $\pi_{\theta}:=\pi_{\theta}(a \mid \mathcal{S}(n))$, which learns the probability of taking action $a \in \mathcal{A}$ given the current state $\mathcal{S}(n)$. An intuitive way to maximize the trajectory reward is to select the optimal action $\mathcal{A}(n)$ in each time interval. However, since the action $\mathcal{A}(n)$ changes the network state, it can potentially affect the rewards in all subsequent time intervals. Therefore, to find a globally optimal UAVBSN deployment, we would like to estimate the utility of an action in terms of maximizing the reward in all future time intervals. To this end, we define the quality of a state-action pair as its expected, cumulative, discounted reward

$$
Q_{\pi}(\mathcal{S}(n), \mathcal{A}(n))=E_{\pi}\left[\sum_{i=n}^{N} \gamma^{i-n} \mathcal{R}(n)\right],
$$

where $\gamma \in(0,1)$ is the discount factor that trades-off immediate rewards with the rewards generated in future time intervals. Taking the expectation over $\pi$ indicates that all subsequent actions (till time interval $N$ ) are selected using the policy $\pi$.

\section{Flow-Level Model for UAVBSN}

As mentioned above, we are interested in training our DRL agent using the steady-state probability distributions

$$
\kappa_{m}\left(x_{m}, t, \mathcal{U}_{\mathrm{UAV}}(t)\right):=\operatorname{Pr}\left[X_{m}(t)=x_{m} \mid t, \mathcal{U}_{\mathrm{UAV}}(t)\right] .
$$

For the derivation of the distribution $\kappa_{m}$, we start with the spatial traffic distribution $\sigma(u, t)$, which can be decomposed as $\sigma(u, t)=\lambda(t) \Omega \delta(u, t)$, where $\lambda(t)$ is the overall data flow 
arrival rate within $\mathcal{L}$ and $\delta(u, t)$ is the normalized spatiotemporal distribution of the traffic in $\mathcal{L}$ at time $t$, so that $\int_{\mathcal{L}} \delta(u, t) \mathrm{d} u=1$.

The UAV BS locations $\mathcal{U}_{\text {UAV }}(t)$ and a user association rule define a partition of $\mathcal{L}$ into cells. Given the locations $\mathcal{U}_{\mathrm{UAV}}(t)$, a cell covered by BS $m \in \mathcal{M}$ is defined as a sub-region $\mathcal{L}_{m}\left(\mathcal{U}_{\mathrm{UAV}}(t)\right) \subset \mathcal{L}$. We assume that the user association rule satisfies the following conditions: the cell shapes do not change for a fixed set of UAV BS locations $\mathcal{U}_{\mathrm{UAV}}(t)$, the cells are non-overlapping, i. e., $\mathcal{L}_{m} \cap \mathcal{L}_{n}=\emptyset, \forall m, n \in \mathcal{M}, m \neq n$, and the cells cover the entire region, i. e., $\bigcup_{m \in \mathcal{M}} \mathcal{L}_{m}=\mathcal{L}$. We further describe a time-variant data flow arrival process in cell $\mathcal{L}_{m}$ as a Poisson process with rate

$$
\lambda_{m}\left(t, \mathcal{U}_{\mathrm{UAV}}(t)\right):=\lambda(t) \int_{\mathcal{L}_{m}\left(\mathcal{U}_{\mathrm{UAV}}(t)\right)} \delta(u, t) \mathrm{d} u .
$$

The traffic within the cell $\mathcal{L}_{m}$ is spatially distributed as

$$
\delta_{m}\left(u, t, \mathcal{U}_{\mathrm{UAV}}(t)\right):=\frac{\lambda(t)}{\lambda_{m}\left(t, \mathcal{U}_{\mathrm{UAV}}(t)\right)} \delta(u, t) .
$$

We use queuing-theoretic tools for interference-coupled networks [49] to approximate the interference coupling effect between UAV BSs in a way, so as to utilize simple, productform formulae to describe the probability distribution $\kappa_{m}$ based on average traffic characteristics $\sigma(u, t)$. It can be shown that the following expression is a good approximation for the mean service rate of a UAV BS $m$, i. e., the average rate with which data flows depart from the system:

$$
\begin{aligned}
& \bar{\mu}_{m}\left(t, \mathcal{U}_{\mathrm{UAV}}(t)\right)= \\
& \quad \frac{1}{\Omega}\left[\int_{\mathcal{L}_{m}\left(\mathcal{U}_{\mathrm{UAV}}(t)\right)} \frac{\delta_{m}\left(u, t, \mathcal{U}_{\mathrm{UAV}}(t)\right)}{\sum_{y \in \mathcal{A}_{m}} \zeta_{m}(y, t) c_{m}(u, y)} \mathrm{d} u\right]^{-1},
\end{aligned}
$$

where $\mathcal{A}_{m}$ is the set of all interference scenarios, in which the $m$-th BS is active, and where $\zeta_{m}(y, t)$ is the probability of seeing interference scenario $y$. Recall that $c_{m}(u, y)$ is the maximally achievable data rate defined in (3), which determines the average duration of a served data flow. We refer the interested reader to [49] for elaborate derivations of (14) and $\zeta_{m}(y, t)$.

Now, having long-term arrival and departure rates, $\lambda_{m}\left(t, \mathcal{U}_{\mathrm{UAV}}(t)\right)$ and $\bar{\mu}_{m}\left(t, \mathcal{U}_{\mathrm{UAV}}(t)\right)$, we formulate an approximation for the steady-state probabilities is

$$
\kappa_{m}\left(x_{m}, t, \mathcal{U}_{\mathrm{UAV}}(t)\right) \approx \frac{\left(1-\rho_{m}\right) \rho_{m}^{x_{m}}}{1-\rho_{m}^{D_{m}+1}}
$$

with UAV BS loads

$$
\rho_{m}\left(t, \mathcal{U}_{\mathrm{UAV}}(t)\right)=\frac{\lambda_{m}\left(t, \mathcal{U}_{\mathrm{UAV}}(t)\right)}{\bar{\mu}_{m}\left(t, \mathcal{U}_{\mathrm{UAV}}(t)\right)} .
$$

Finally, two important metrics can be deduced from the steady-state probability distribution $\kappa\left(x, t, \mathcal{U}_{\mathrm{UAV}}(t)\right)$, namely the flow blocking probability $P_{m}\left(t, \mathcal{U}_{\mathrm{UAV}}(t)\right)$ and the average flow throughput (of admitted flows) $R_{m}\left(t, \mathcal{U}_{\mathrm{UAV}}(t)\right)$. The flow blocking probability, essentially, describes the likelihood that an arriving data flow is blocked because the UAV BS serves already $D_{m}$ flows. According to the PASTA property, we have $P_{m}\left(t, \mathcal{U}_{\mathrm{UAV}}(t)\right)=\kappa_{m}\left(D_{m}, t, \mathcal{U}_{\mathrm{UAV}}(t)\right)$. The average flow throughput is defined as the expected ratio of the flow size (in Mbps) and the time the flow is active. The expectation refers to the fluctuations of the interference and of the number of competing data flows in the same cell and the different receiving conditions across a cell area. For brevity, we refer to [49] and Eqs. (16)-(21) therein for a more elaborate derivation.

For given radio propagation environment, steady-state user traffic densities, and the UAV BS locations $\mathcal{U}_{\mathrm{UAV}}(n)$, the FLM provides the steady-state probability distribution,

$$
\begin{aligned}
\mathcal{P}(n):=\left[\kappa_{1}(0), \ldots, \kappa_{1}\left(D_{1}\right), \ldots,\right. & \\
& \left.\kappa_{M}(0), \ldots, \kappa_{M}\left(D_{M}\right),\right]\left(t_{n}, \mathcal{U}_{\mathrm{UAV}}(n)\right),
\end{aligned}
$$

where $t_{n}$ is the beginning of the $n^{\text {th }}$ time interval. Further, the FLM also provides the corresponding reward

$\mathcal{R}(n):=\sum_{m \in \mathcal{M}} \log \left[\left(1-P_{m}\left(t_{n}, \mathcal{U}_{\mathrm{UAV}}(n)\right)\right) R_{m}\left(t_{n}, \mathcal{U}_{\mathrm{UAV}}(n)\right)\right]$

where the first product term in the log is the non-blocking probability for the $m^{\text {th }}$ UAV BS, and the second product term is the average steady-state flow throughput experienced by a data flow served by the same UAV BS. Maximizing this reward function therefore is equivalent to balancing the traffic loads across the UAV BSs so that fewer flows are blocked, while simultaneously attempting to increase the network throughput.

\section{Learning the Optimal Policy}

Recall that the goal of the trajectory optimization policy is to maximize the cumulative reward defined in (9), which can be decomposed as the problem of finding the state-action pair with the highest quality function defined in 10 . In general, the quality function can be calculated using the flow-level model and the optimal policy can subsequently be obtained using dynamic programming. However in each time interval, the space of actions for $M$ UAV BSs spans $M^{7}$ distinct possibilities. Consequently, for a trajectory spanning $N$ time intervals, there exist $M^{7 N}$ actions. For a realistic scenario with $M=9 \mathrm{UAV}$ BSs and $N=20$, the policy space comprises $9^{140}$ actions, which is infeasible to enumerate in real time.

We propose DRL to learn the trajectory optimization policy through random exploration of the action space and iteratively updating the learnt policy. The training proceeds as follows: For an observed state $\mathcal{S}(n)$ at the end of time interval $n$, the DRL agent provides a probability distribution over the action space, which is sampled to predict the next action $\mathcal{A}(n)$. Then, the FLM is queried to obtain the steady-state reward $\mathcal{R}(n)$ and the next state $\mathcal{S}(n+1)$. The tuple $(\mathcal{S}(n), \mathcal{A}(n), \mathcal{R}(n))$ is appended to the training data, and the next time interval is processed. A training episode is defined as the set of all training samples during $n \in \mathcal{N}$. We use the training dataset to iteratively train the DRL agent. Several DRL schemes are 
available training; we refer the reader to [50] for a timely exposition on this subject.

For an input network state, our proposed DRL agent generates a probability distribution over the available actions. This mapping from an input network state to the probability distribution over the actions is modeled using a deep artificial neural network, which is known to be a good approximator for high-dimensional non-linear functions. Since the DRL agent directly learns the action policy, our approach falls under the class of policy gradient techniques developed over the last decade [50]. Policy gradient techniques work by iteratively updating the policy parameters through stochastic gradient updates, where the gradient updates are generated using the training dataset described above. However, since policy gradient methods use probabilistic selection of the available actions, if a few particularly bad actions get selected, they are known to suffer from large gradients and consequently, poor training.

Recently, trust region policy optimization (TRPO) have been developed to mitigate the impact of randomly selecting poor actions [51]. These techniques adjust the gradient updates with a carefully constructed weighting rule that dampens the effect of actions that have a small selection probability from the DRL agent. In this paper, we use the state-of-theart TRPO approach, known as proximal policy optimization (PPO), which uses gradient clipping to make sure that poor actions do not disrupt the training [52]. PPO achieves this by calculating the gradient of the policy parameters using the rule

$$
\begin{aligned}
& \nabla_{\theta}= \arg \max _{\theta}\left(E_{n}\left[r_{\theta}(n) \hat{A}(n), \operatorname{clip}\left(r_{\theta}(n), 1-\epsilon, 1+\epsilon\right) \hat{A}(n)\right]\right), \\
& r_{\theta}(n)=\frac{\pi_{\theta}(\mathcal{A}(n) \mid \mathcal{S}(n))}{\pi_{\theta_{\text {old }}}(\mathcal{A}(n) \mid \mathcal{S}(n))} \\
& \hat{A}(n)=Q_{\pi}(\mathcal{S}(n), \mathcal{A}(n))-\sum_{a \in \mathcal{A}} \pi_{\theta}(a \mid \mathcal{S}(n)) Q_{\pi}(\mathcal{S}(n), a)
\end{aligned}
$$

where $\hat{A}(n)$ is the advantage function that quantifies the benefit taking action $\mathcal{A}(n)$ over the set of available actions. The advantage function relies on the quality function defined in (10). A complete description of PPO is rather involved, and we refer the interested reader to [52] for the details.

Our proposed DRL approach for learning the trajectory optimization policy satisfies our learning goals of being fast, efficient and robust: (i) We directly learn the trajectory optimization policy through stochastic gradient updates of the policy parameters, which results in fast convergence to optimum, (ii) During the random exploration phase of the policy training, we use the FLM model to estimate the rewards for randomly selected actions. Since the training data is generated offline, the learning is far more efficient compared to executing those actions online in a simulator or in the real world. (iii) We use a carefully defined, cumulative reward metric over the entire trajectory, which ensures that the learnt policy is robust in terms of the performance in each time interval.

\section{Numerical Results}

In the numerical results that follow, we demonstrate UAV trajectory optimization in an urban environment for two key traffic scenarios: uniform and non-uniform user traffic densities respectively. We choose the user traffic distribution to be lognormally distributed [53], [54] and spatially correlated with correlation distance $d_{\mathrm{c}}$ and consider outdoor traffic only. In addition to the correlation distance, the traffic distribution is also characterized by its mean $\tau$ and its standard deviation $\nu$. In the first scenario, we assume that the user traffic is distributed uniformly over the served region with a mean of $\tau=100 \mathrm{Mbps} / \mathrm{km}^{2}$ and $\nu=0$. In this case, to optimize the network performance, the UAVs need to account for the radio propagation characteristics of the region. Consequently for this scenario, the DRL agent attempts to learn UAV trajectories that efficiently navigate the topography of the urban area. In the second scenario, we assume the the user traffic distribution follows a mean of $\tau=100 \mathrm{Mbps} / \mathrm{km}^{2}$ and a standard deviation of $\nu=50$. This non-uniform user density leads to the presence of traffic hotspots within the served region. Therefore, the learnt UAV trajectories must account for both the propagation environment as well as the hotspots that are distributed randomly within the served area. Since the location of traffic hotspots is not directly observable and is instead stochastically captured by the observed network state (6), this scenario constitutes a more difficult learning task.

State-of-the-art approaches propose optimal UAV placement based on the circle packing theory [55], [56]. For a given user traffic load and a uniform UAV height above the served region, circle-packed placement maximizes the coverage area. Consequently, we use the circle packing theory for determining the horizontal coordinates of the UAVs. Since we study an urban environment with a non-uniform elevation profile, the initial vertical placement of the UAVs is chosen to be at the same distance from each UAV to the surface, i.e. top of a building or street. Starting with this initial deployment, the DRL attempts to learn the optimal UAV trajectories that maximizes the network performance objective described in 9 for the reward metric defined in (18).

\section{A. Evaluation Framework}

We test our proposed approach for UAV trajectory optimization in an urban environment. In particular, we use the threedimensional model of a section of Downtown San Francisco, whose building height distribution is depicted in Fig.2 2. The scenario is a heavily built-up urban area with a complex radio environment characterized by non-line-of-sight propagation as well as street canyons. We obtained this model by using the OpenStreetMap plugin v2.3.9 [57] of Blender v2.79b [58], and imported the height map into MATLAB, which is then used for FLM as well as the simulation environment.

The FLM is implemented in MATLAB and is configured with the spatial traffic distribution, the topographical map of the served area, and the dynamic UAV BS locations. We 


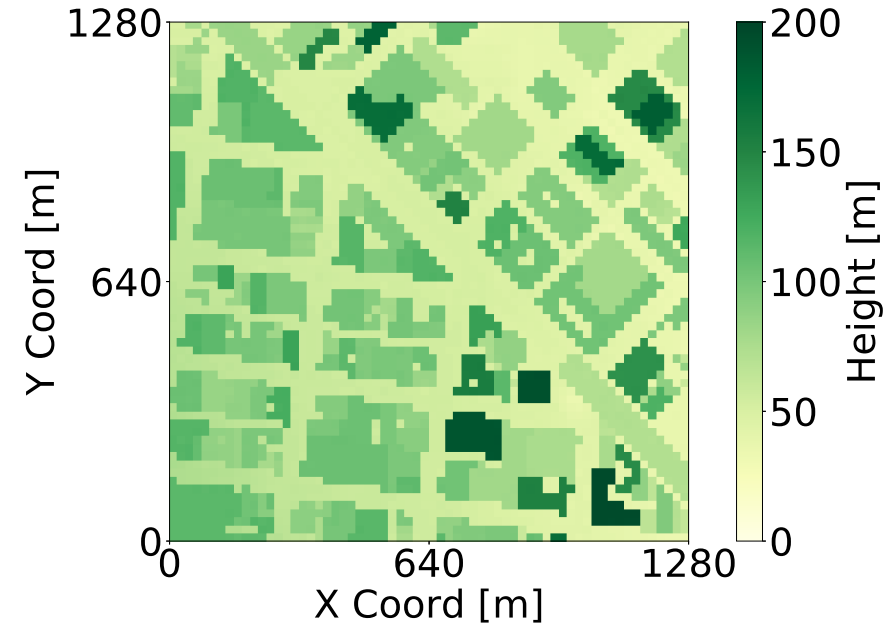

Fig. 2. Elevation map for the Downtown San Francisco scenario considered here. The map is discretized into $64 \times 64$ square pixels spanning $20 \mathrm{~m}$ in each direction. Buildings are visible as the darker shaded regions.

encapsulate the FLM within a Python-based OpenAI Gym environment [59], which provides a standard interface for executing DRL-based applications. Using this FLM interface, we a DRL agent to learn a neural network model of the optimal UAV trajectories. The neural network is conveniently and efficiently implemented using the Tensorflow package [60]. Further, we use an off-the-shelf implementation of the PPO algorithm available as a part of the Ray RLLib library [61]. By parallelizing several random instantiations of the FLM over a computational cluster, RLLib enables the time-efficient generation of training episodes that are used for learning.

The actual numerical evaluation is then carried out with a discrete event simulator, which is implemented in MATLAB and which is based on the general system model presented in Section III . We test the DRL agent for predicting the UAV trajectories using the discrete event simulator. This simulation environment relies on the same topographical city model as the FLM. However, while the FLM only predicts the steady-state network metrics, the simulation environment explicitly models the stochastic flow arrival, service and departure processes. For each of the $N=20$ UAV BS trajectory steps, a simulation with a fixed duration of $3 \mathrm{~min}$ is carried out, in which the empirical probability distribution of $X(t)$ is recorded and used to produce UAV movement actions triggered by the trained agent. Each step carries over the previous network state (active flows), which might result in blocking of flows if they are handed over to an already full UAV BS. Further for the non-uniform traffic scenario, the traffic hotspot locations are generated independently for the FLM and simulation runs.

We consider a rectangular region served by 9 UAV BSs. For this configuration, the circle packing theory predicts horizontal UAV locations arranged on a regular $3 \times 3$ grid [62]. Further, we associate each user to the UAV BS that has the highest received signal power at the location of the user. The model parameters, simulator settings, and DRL hyper-parameters are listed in Tables II II, and III, respectively.
TABLE I

MODEL PARAMETERS

\begin{tabular}{ll}
\hline Parameter & Value \\
\hline Number $M$ of UAV BSs & 9 \\
Maximum number $D_{m}$ of flows & 10 \\
Number $N$ of trajectory steps & 20 \\
Horizontal step size $\Delta u_{h}$ & $40 \mathrm{~m}$ \\
Horizontal step size $\Delta u_{v}$ & $20 \mathrm{~m}$ \\
Traffic correlation distance $d_{\mathrm{c}}$ & $60 \mathrm{~m}$ \\
Antenna pattern $A_{m}$ & Omni-directional \\
MIMO diversity gain $D_{\mathrm{MIMO}}$ & $3 \mathrm{~dB}$ \\
Fast fading margin $M_{\mathrm{FF}}$ & $2 \mathrm{~dB}$ \\
Transmitter noise figure $N F_{\mathrm{BS}}$ & $5 \mathrm{~dB}$ \\
Receiver noise figure $N F_{\mathrm{UE}}$ & $9 \mathrm{~dB}$ \\
UAV LOS attenuation $N L$ [42] & $1.6 \mathrm{~dB}$ \\
UAV NLOS attenuation $N L$ 142] & $23 \mathrm{~dB}$ \\
Carrier frequency $f_{\mathrm{c}}$ & $2 \mathrm{GHz}$ \\
Noise spectral density $N_{0}$ & $-174 \mathrm{dBm} / \mathrm{Hz}$ \\
System bandwidth $B$ & $20 \mathrm{MHz}$ \\
Maximum achievable rate $c_{\mathrm{max}}$ & $6 \mathrm{bps} / \mathrm{Hz}(64-\mathrm{QAM})$ \\
Mean flow size $\Omega$ & $2 \mathrm{Mbit}$ \\
Transmit power $p_{\mathrm{tx}, m}$ & $37 \mathrm{dBm}$ \\
\hline
\end{tabular}

TABLE II

Discrete Event Simulator SetTings

\begin{tabular}{ll}
\hline Parameter & Value \\
\hline Scheduler & Round Robin \\
Arrival process & Poissonian \\
Flow size distribution & Exponential \\
Simulation time interval per trajectory step & 3 min \\
\hline
\end{tabular}

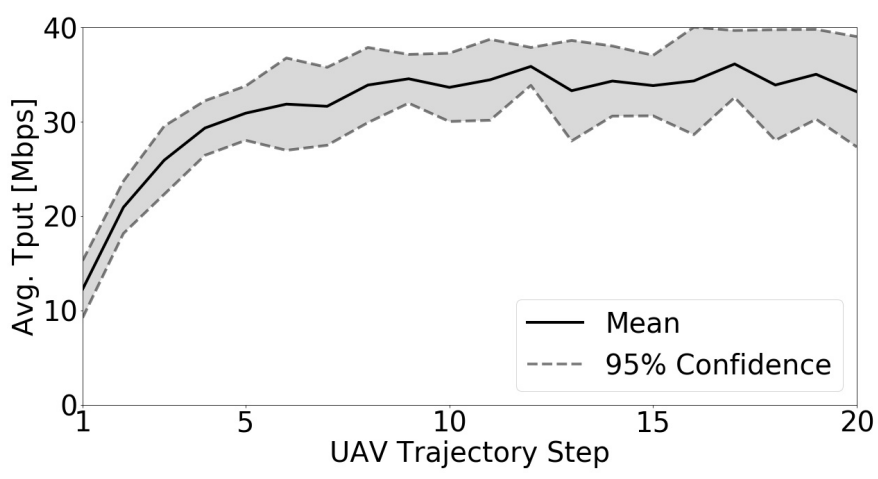

Fig. 3. Average flow throughput versus the trajectory step for uniform traffic density with mean $\tau=100 \mathrm{Mbps} / \mathrm{km}^{2}$. The proposed approach increases the throughput by approximately $300 \%$ over the initial circle-packed deployment.

\section{B. Simulation Results}

Fig. 4 depicts example trajectories of the UAV BSs for uniform traffic density scenario and four independent test runs. It can be seen that some UAV BSs more or less hover around their initial locations and other UAV BSs change locations quite actively, flying distances of multiple hundred meters. Further, the trajectories are similar across the test runs, as expected for a uniform user traffic. The small trajectory differences arise from the stochastic traffic fluctuations, which leads to different actions suggested by the trained agent. From Fig. 3, it becomes apparent that the UAV movements lead to increased, average flow throughput as the UAVs execute the trajectory. The solid black line indicates the average flow throughput for the network, obtained by the discrete event simulation, 
TABLE III

DRL HYPER-PARAMETERS

\begin{tabular}{ll}
\hline Parameter & Value \\
\hline Neural Network layout & Fully Connected \\
Neural Network layer sizes & {$[256,128,64,128,256]$} \\
Neural Network activation function & ReLU \\
Learning scheme & PPO \\
Learning rate & 0.0005 \\
Mini-batch size & 8 \\
SGD iterations & 32 \\
Clip parameter, $\epsilon$ & 0.3 \\
Training epochs. & $2.5 \times 10^{6}$ \\
\hline
\end{tabular}
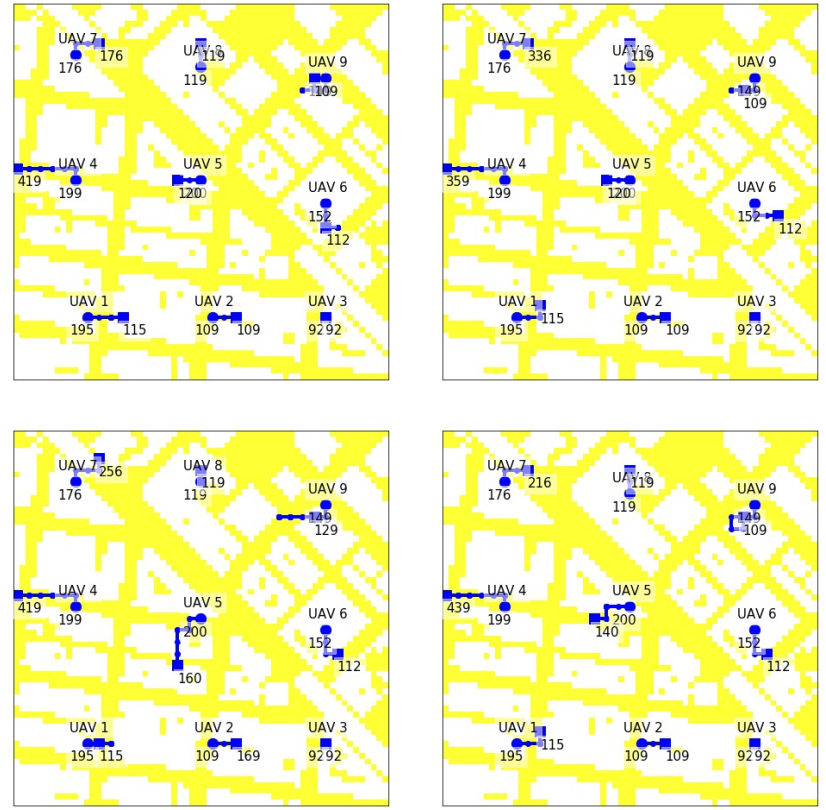

Fig. 4. UAV trajectories for uniform traffic density with mean $\tau=$ $100 \mathrm{Mbps} / \mathrm{km}^{2}$ and four random traffic realizations. Solid circles and squares denote UAV start and end positions and the numerical value denote their respective heights (in meters). The trajectories are similar, with slight differences arising due from the stochastic traffic variations.

for 32 test runs. The $95 \%$ confidence interval is indicated as the gray shaded region, which is again a consequence of stochastic flow arrivals and flow sizes. Nevertheless, the trend of increasing average throughput with the training iteration (not shown here for brevity) indicates that the optimization is largely robust against random traffic fluctuations. Fig. 3 also reveals that there is a quite sharp increase of performance for in the first 5 steps executed by the UAVs, which then saturates to some extent. The relative gain of the average flow throughput is approximately a factor of three for the entire UAV trajectory. Finally, Fig. 5 shows the empirical probability distributions of the nine UAV BSs (nine bars per diagram) at their initial locations (four left diagrams) and at their final locations (four right diagrams) and for the four test trajectories (four pairs of diagrams). The coloring of the bars indicate the individual probability distribution per UAV BS, where light blue refers to zero active flows (UAV BS inactivity) and dark red $(x=10)$ indicates the amount of blocked flows. We observe that heavily loaded UAV BSs are able to reduce
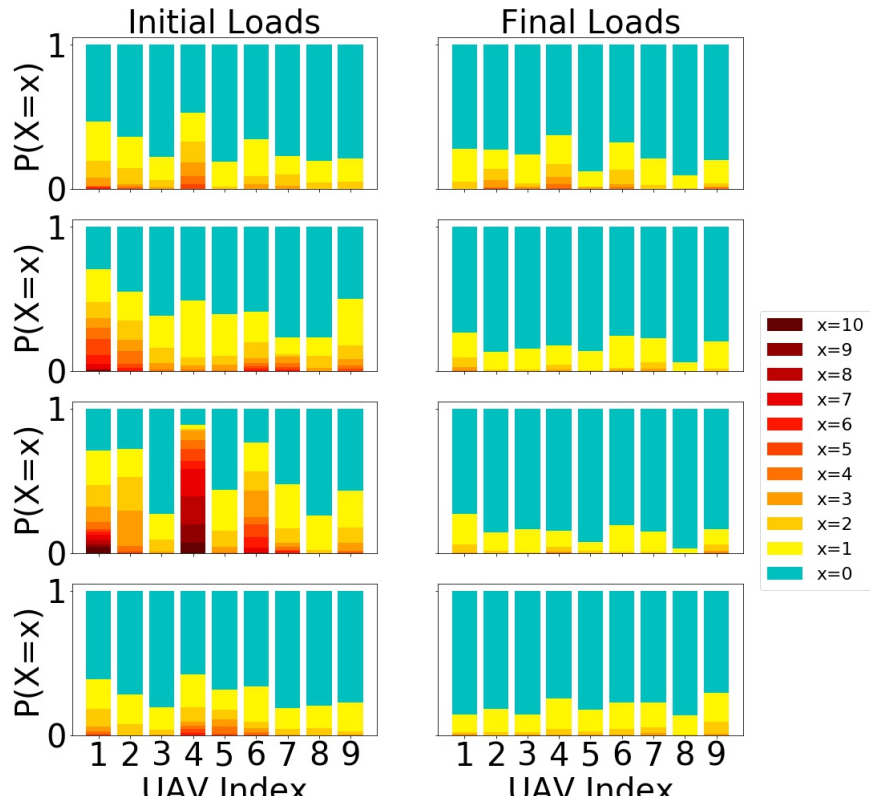

Fig. 5. Empirical distribution of the load on each UAV BS for uniform traffic density with mean value $\tau=100 \mathrm{Mbps} / \mathrm{km}^{2}$ and four random traffic realizations. The served traffic load is illustrated at the starting UAV locations (left plots) and the final UAV locations (right plots).

their load considerably, leading to a well balanced situation at the end of the simulation. From Fig. 4, we see that this is achieved by UAV 4 ascending and effectively covering less traffic and UAVs 1 and 6 descending into street canyons, which improves their probability of line-of-sight with served users while simultaneously reducing interference to other UAVs. The empirical simulation results at the end of the UAVs trajectories also indicate that the overall load of the system is decreased substantially, which results from a by far more relaxed interference situation among the UAV BSs and, hence, increased spectral efficiency. The similarity of the resulting empirical distribution among the different trajectories is also an indicator for the robustness of the approach.

The non-uniform traffic density scenario is more challenging as, due to the presence of hotspots, most of the UAV BSs are heavily loaded or overloaded. Fig.77 shows the UAV trajectories for four test runs with hotspots. It can be seen that UAV trajectories are more diverse as compared to the uniform traffic scenario. However, the trajectories still retain some amount of similarity which arises from exploiting the radio propagation environment generated by the topography of the studied scenario. UAVs 2 and 5, show substantial differences in their trajectories across the test runs, even moving in opposing directions depending on the location of the hotspots. UAV 4 ascends in order to reduce interference and distribute the load more evenly across the network. UAVs 1, 6, and 3 exploit street canyons for better radio propagation conditions. The favorable effects of sharing traffic by descending and of divergent trajectories are attributed to the FLMs ability to explicitly take into account the loads of the UAV BSs and intercell interference dynamics, respectively. In particular, already five UAV steps lead to tripling of the average flow throughput, 


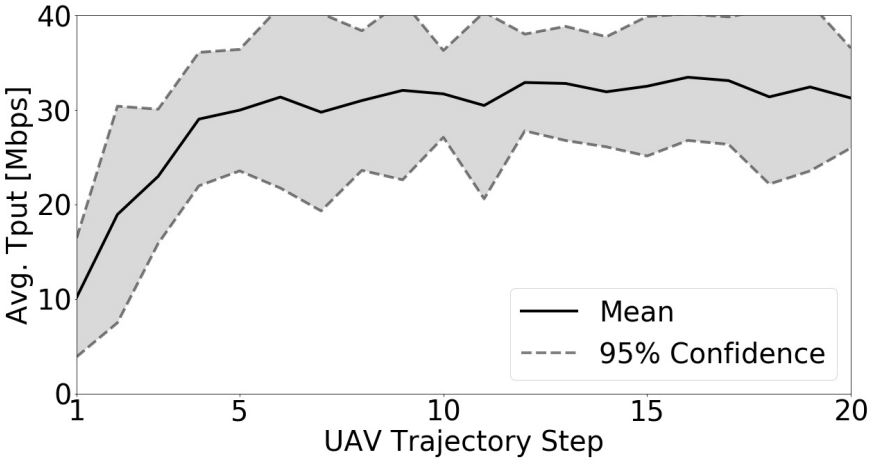

Fig. 6. Average flow throughput versus the trajectory step for non-uniform traffic density with mean $\tau=100 \mathrm{Mbps} / \mathrm{km}^{2}$ and standard deviation $\nu=50$. The proposed trajectory optimization approach increases the throughput by approximately $300 \%$ over the initial circle-packed deployment.

which reaches almost $30 \mathrm{Mbps}$ towards the end of the UAV trajectory (again a three-fold increase in performance). It also becomes apparent from the increasing trend of the performance around the mean (blue curve) that the robustness of the algorithm against random hotspot locations is sufficiently high. Finally, Fig. 8 depicts the empirical probability distributions of concurrently active flows for each UAV BS at the beginning and at the end of the four test trajectories. For all four example text runs, the overload situation is substantially relieved. While at least five UAV BSs are in overload (probability of zero active flows close to zero) and two more are heavily loaded at the beginning of the optimization, only two or three UAV BSs are moderately loaded and the rest are lightly loaded at the end of optimization, leading to a well-balanced network. Similar to the uniform traffic density scenario, the average throughput is simultaneously improved. Nevertheless, the algorithm has its limits as the network load has the possibility to be made more even. It can be assumed that the current maximum UAV travel distance and the number of training iterations are limiting factors here.

\section{Discussion}

The simulation results show that the DRL approach converges to optimal policies by training on the FLM, in terms of increasing average flow throughputs while simultaneously balancing traffic loads across the UAV BS. For our choice of hyper-parameters, selected by carefully searching though a grid of possible values, the DRL agent can be trained at time-scales comparable to the user traffic variations by distributed exploration over several, parallel, FLM instances. Further, the FLM-based approach is efficient, which can be seen by rather directed UAV trajectories in most cases. We also notice that it has robustness against random traffic statistics, which attributed to the fact that, with the chosen settings, three minutes time for gathering empirical scheduling information for each UAV location. Using this information in each step, the network is able to optimize trajectories spanning twenty steps, which corresponds to sixty minutes of serving traffic. Furthermore, assuming that spatial traffic distributions change
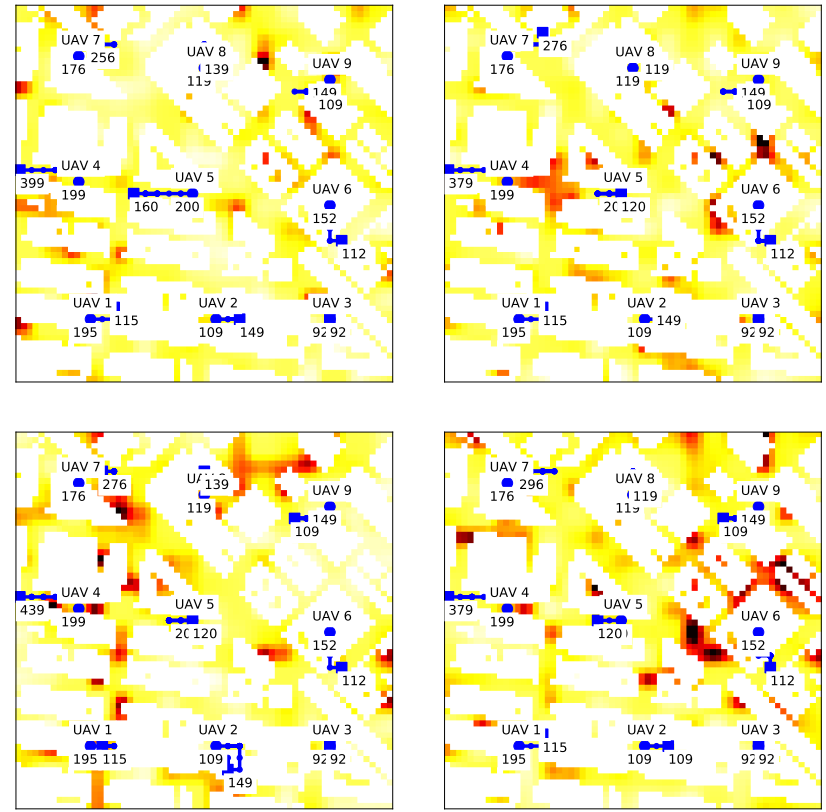

Fig. 7. UAV trajectories for non-uniform traffic density with mean $\tau=$ $100 \mathrm{Mbps} / \mathrm{km}^{2}$ and standard deviation $\nu=50 \mathrm{Mbps} / \mathrm{km}^{2}$, and four random traffic realizations. Solid circles and squares denote UAV start and end positions and the numerical value denote their respective heights (in meters). The trajectories vary substantially across the test runs from some UAVs, depending on the location of the traffic hotspots.
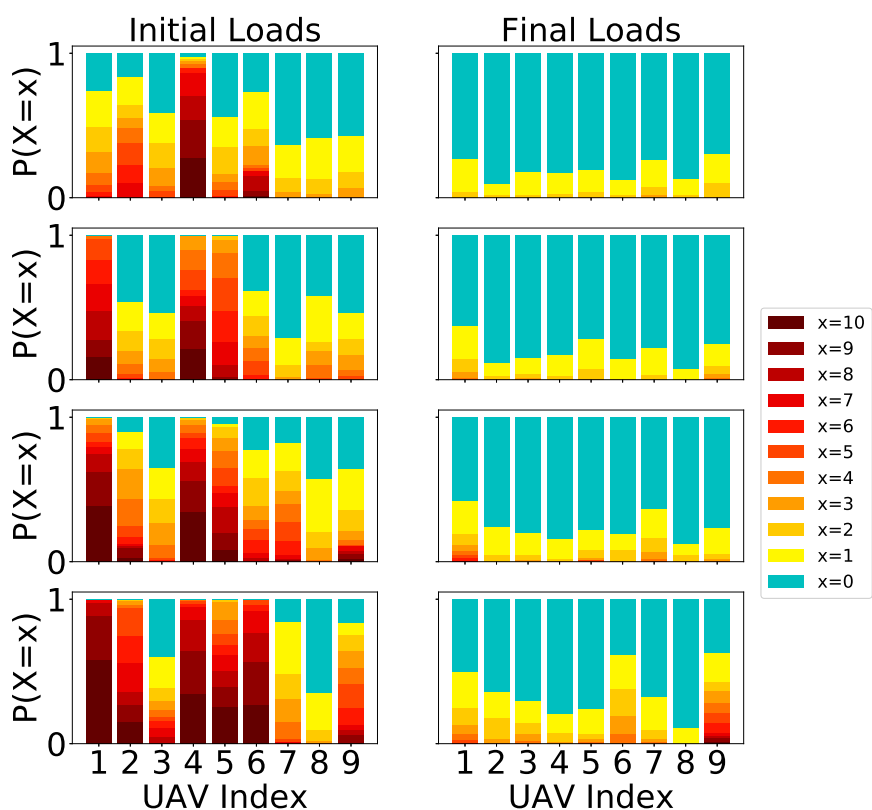

Fig. 8. Empirical distribution of the load on each UAV BS for non-uniform traffic density with mean value $\tau=100 \mathrm{Mbps} / \mathrm{km}^{2}$ and standard deviation $\nu=50 \mathrm{Mbps} / \mathrm{km}^{2}$, and four random traffic realizations. The served traffic load is illustrated at the starting UAV locations (left plots) and the final UAV locations (right plots).

relatively slowly over the course of a day compared to the optimization intervals, the algorithm is fast enough to lead to a substantial system performance improvement (up to a fourfold increase of the average throughput experienced by a flow). 


\section{CONCLUSion AND Future Work}

In this paper, we have proposed FLM for the realistic modeling of UAVBSNs. Our model incorporates user traffic distributions and complex radio propagation environments. Further, we have proposed a DRL approach to learn optimal UAV BS actions that efficiently navigates the extremely high-dimensional action space. Our DRL approach exploits the FLM for fast, efficient, and robust learning of UAV BS trajectories for a target objective that balances the load across the UAV BSs while rewarding higher network spectral efficiency. Future work on FLM-based DRL algorithms for UAVBSN would include considering spatial traffic distributions that vary on the same time scale as UAV BS movements. Here, the agent needs to learn not only the relation of steady-state (or empirical) distributions to the resulting reward but also how the reward changes in future time steps given typical daily variations of the traffic in time and space. In addition, the current study takes into account only the same starting positions for the UAVs. It is unclear how the current implementation is able to generalize towards arbitrary starting positions. We anticipate that the agent would requires additional spatial information to learn the system behavior in such a case.

\section{ACKNOWLEDGMENT}

This work was supported by a fellowship within the FITweltweit programme of the German Academic Exchange Service (DAAD). This work was partially supported by the Wallenberg AI, Autonomous Systems and Software Program (WASP) funded by the Knut and Alice Wallenberg Foundation. We thank the anonymous reviewers for their extensive feedback that has helped in substantially improving the paper.

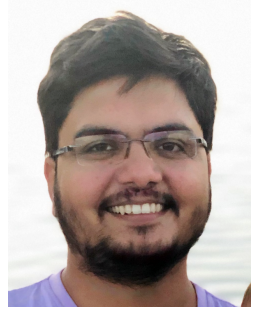

Vidit Saxena Vidit Saxena received his B. Tech. in Electronice and Comminication Engineering from the Indian Institute of Technology (IIT), Roorkee, in 2009, and his M. Sc. in Wireless Communication from Faculty of Engineering (LTH), Sweden, in 2014. Since August 2014, he has been with Ericsson Research, Sweden, as a researcher at their radio department where he contributed to the standardization of multi-antenna protocols and machine-type communications in $4 \mathrm{G}$ and $5 \mathrm{G}$ networks. In February 2016, he was accepted into his current industrial doctoral position at the Royal Institute of Technology (KTH), Sweden with scholarship from the Wallenberg Artificial Intelligence, Autonomous Systems and Software Program (WASP), Sweden. In his current position, Vidit investigates machine learning techniques for physical layer optimization in cellular networks. During Fall 2018 and Summer 2019, he was a visiting scholar at RISELab, University of California, Berkeley, USA.

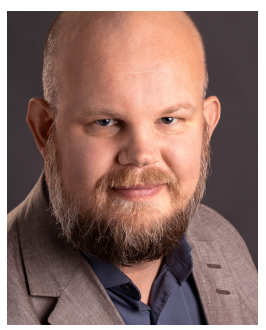

Joakim Jaldén oakim Jaldénoakim JaldénJ (S'03M'08-S'13) received the M.Sc. and Ph.D. in electrical engineering from the Royal Institute of Technology (KTH), Stockholm, Sweden in 2002 and 2007 respectively. From July 2007 to June 2009 he held a post-doctoral research position at the Vienna University of Technology, Vienna, Austria. He also studied at Stanford University, CA, USA, from September 2000 to May 2002, and worked at ETH, Zürich, Switzerland, as a visiting researcher, from August to September, 2008. In July 2009 he returned to $\mathrm{KTH}$, where he is now a professor of signal processing. He was an associate editor for the IEEE Communications Letters between 2009 and 2011, and an associate editor for the IEEE Transactions in Signal Processing between 2012 and 2016. He has been a member of the IEEE Signal Processing for Communications and Networking Technical Committee (SPCOM-TC) since 2013, where he serves as chair 2019-2020. Since 2016 he is also responsible for the five year B.Sc and M.Sc. Degree Program in Electrical Engineering at $\mathrm{KTH}$.

For his work on MIMO communications, Joakim has been awarded the IEEE Signal Processing Society's 2006 Young Author Best Paper Award, and the best student conference paper award at IEEE ICASSP 2007. He was also a recipient of the Ingvar Carlsson Career Award issued in 2009 by the Swedish Foundation for Strategic Research. His recent work includes work on signal processing for biomedical data analysis, including single-cell tracking for time-lapse microscopy and inverse diffusion for immunoassays.

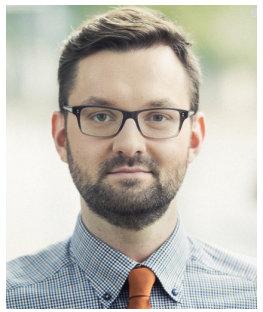

Henrik Klessig Henrik Klessig received his $M$. Sc. (Dipl.-Ing.) in Information Systems Engineering and his Ph. D. (Dr.-Ing.) in Electrical Engineering from Technical University Dresden, Germany, in 2012 and 2016, respectively. He was a research intern at Bell Labs, Stuttgart, Germany, in 2010 and a research associate at the Vodafone Chair, TU Dresden between 2012 and 2016, where he has been working on queuing and traffic theory and its application to self-organizing networks. He was a recipient of the FITweltweit postdoc stipend from the German Academic Exchange Service and has been working on edge computing service continuity and aerial wireless networks at the International Computer Science Institute. He was also a visiting researcher at UC Berkeley in 2018. Since December 2018, he has been with Robert Bosch GmbH in Renningen, Germany. 


\section{REFERENCES}

[1] B. Vergouw, H. Nagel, G. Bondt, and B. Custers, Drone Technology: Types, Payloads, Applications, Frequency Spectrum Issues and Future Developments. The Hague: T.M.C. Asser Press, 2016, pp. 21-45. [Online]. Available: https://doi.org/10.1007/978-94-6265-132-6_2

[2] S. D. Muruganathan, X. Lin, H. Maattanen, Z. Zou, W. A. Hapsari, and S. Yasukawa, "An overview of 3gpp release-15 study on enhanced LTE support for connected drones," CoRR, vol. abs/1805.00826, 2018. [Online]. Available: http://arxiv.org/abs/1805.00826

[3] C. T. Cicek, H. Gultekin, B. Tavli, and H. Yanikomeroglu, "Uav base station location optimization for next generation wireless networks: Overview and future research directions," in 2019 1st International Conference on Unmanned Vehicle Systems-Oman (UVS), Feb 2019, pp. $1-6$.

[4] T. Tozer and D. Grace, "High-Altitude Platforms for Wireless Communications," Electronics \& Communication Engineering Journal, vol. 13, pp. 127-137(10), June 2001. [Online]. Available: http: //digital-library.theiet.org/content/journals/10.1049/ecej_20010303

[5] "Balloon-powered Internet for everyone". Project Loon. [Online]. Available: https://x.company/loon/

[6] Y. Zeng, R. Zhang, and T. J. Lim, "Wireless Communications with Unmanned Aerial Vehicles: Opportunities and Challenges," IEEE Communications Magazine, vol. 54, no. 5, pp. 36-42, May 2016.

[7] I. Bor-Yaliniz and H. Yanikomeroglu, "The New Frontier in RAN Heterogeneity: Multi-Tier Drone-Cells," IEEE Communications Magazine, vol. 54, no. 11, pp. 48-55, November 2016.

[8] M. Alzenad, M. Z. Shakir, H. Yanikomeroglu, and M. S. Alouini, "FSO-Based Vertical Backhaul/Fronthaul Framework for 5G+ Wireless Networks," IEEE Communications Magazine, vol. 56, no. 1, pp. 218 224, Jan 2018.

[9] S. Sekander, H. Tabassum, and E. Hossain, "Multi-Tier Drone Architecture for 5G/B5G Cellular Networks: Challenges, Trends, and Prospects," IEEE Communications Magazine, vol. 56, no. 3, pp. 96-103, MARCH 2018.

[10] A. Al-Hourani, S. Kandeepan, and A. Jamalipour, "Modeling Air-toGround Path Loss for Low Altitude Platforms in Urban Environments," in 2014 IEEE Global Communications Conference, Dec 2014, pp. 28982904.

[11] J. Garza, M. Panduro, A. Reyna, G. Romero, and C. del Rio, "Design of UAVs-Based 3D Antenna Arrays for a Maximum Performance in Terms of Directivity and SLL," International Journal of Antennas and Propagation, 2016.

[12] M. Mozaffari, W. Saad, M. Bennis, and M. Debbah. "Drone-based Antenna Array for Service Time Minimization in Wireless Networks". [Online]. Available: http://www.laneas.com/publication/ drone-based-antenna-array-service-time-minimization-wireless-networks

[13] M. Mozaffari, W. Saad, M. Bennis, and M. Debbah, "Communications and control for wireless drone-based antenna array," IEEE Transactions on Communications, vol. 67, no. 1, pp. 820-834, 2018.

[14] S. Jeong, O. Simeone, and J. Kang, "Mobile edge computing via a uavmounted cloudlet: Optimization of bit allocation and path planning," IEEE Transactions on Vehicular Technology, vol. 67, no. 3, pp. 20492063, March 2018.

[15] F. Tang, Z. M. Fadlullah, B. Mao, N. Kato, F. Ono, and R. Miura, "On a novel adaptive uav-mounted cloudlet-aided recommendation system for lbsns," IEEE Transactions on Emerging Topics in Computing, pp. 1-1, 2018.

[16] V. Mnih, K. Kavukcuoglu, D. Silver, A. A. Rusu, J. Veness, M. G Bellemare, A. Graves, M. Riedmiller, A. K. Fidjeland, G. Ostrovski et al., "Human-level control through deep reinforcement learning," Nature, vol. 518, no. 7540, p. 529, 2015.

[17] D. Silver, A. Huang, C. J. Maddison, A. Guez, L. Sifre, G. Van Den Driessche, J. Schrittwieser, I. Antonoglou, V. Panneershelvam, M. Lanctot et al., "Mastering the game of go with deep neural networks and tree search," nature, vol. 529, no. 7587, p. 484, 2016.
[18] S. Gu, E. Holly, T. Lillicrap, and S. Levine, "Deep reinforcement learning for robotic manipulation with asynchronous off-policy updates," in 2017 IEEE International Conference on Robotics and Automation (ICRA). IEEE, 2017, pp. 3389-3396.

[19] J. Lyu, Y. Zeng, R. Zhang, and T. J. Lim, "Placement optimization of uav-mounted mobile base stations," IEEE Communications Letters, vol. 21, no. 3, pp. 604-607, 2017.

[20] R. Ghanavi, E. Kalantari, M. Sabbaghian, H. Yanikomeroglu, and A. Yongacoglu, "Efficient 3d aerial base station placement considering users mobility by reinforcement learning," in 2018 IEEE Wireless Communications and Networking Conference (WCNC). IEEE, 2018, pp. 1-6.

[21] Y. Zeng and R. Zhang, "Energy-efficient uav communication with trajectory optimization," IEEE Transactions on Wireless Communications, vol. 16 , no. 6 , pp. $3747-3760,2017$.

[22] S. Zhang, Y. Zeng, and R. Zhang, "Cellular-enabled uav communication: Trajectory optimization under connectivity constraint," in 2018 IEEE International Conference on Communications (ICC). IEEE, 2018, pp. $1-6$.

[23] Q. Wu, Y. Zeng, and R. Zhang, "Joint trajectory and communication design for multi-uav enabled wireless networks," IEEE Transactions on Wireless Communications, vol. 17, no. 3, pp. 2109-2121, 2018.

[24] F. Cheng, S. Zhang, Z. Li, Y. Chen, N. Zhao, F. R. Yu, and V. C. Leung, "Uav trajectory optimization for data offloading at the edge of multiple cells," IEEE Transactions on Vehicular Technology, vol. 67, no. 7, pp. 6732-6736, 2018

[25] R. Gangula, P. de Kerret, O. Esrafilian, and D. Gesbert, "Trajectory optimization for mobile access point," in 2017 51st Asilomar Conference on Signals, Systems, and Computers. IEEE, 2017, pp. 1412-1416.

[26] R. Gangula, D. Gesbert, D. F. Külzer, and J. M. Franceschi, "A landing spot approach for enhancing the performance of uav-aided wireless networks," in 2018 IEEE International Conference on Communications Workshops (ICC Workshops). IEEE, 2018, pp. 1-6.

[27] H. Bayerlein, P. De Kerret, and D. Gesbert, "Trajectory optimization for autonomous flying base station via reinforcement learning," in 2018 IEEE 19th International Workshop on Signal Processing Advances in Wireless Communications (SPAWC), June 2018, pp. 1-5.

[28] M. Mozaffari, W. Saad, M. Bennis, and M. Debbah, "Drone Small Cells in the Clouds: Design, Deployment and Performance Analysis," CoRR, vol. abs/1509.01655, 2015. [Online]. Available: http://arxiv.org/abs/1509.01655

[29] — "Optimal Transport Theory for Power-Efficient Deployment of Unmanned Aerial Vehicles," in 2016 IEEE International Conference on Communications (ICC), May 2016, pp. 1-6.

[30] R. Ghanavi, E. Kalantari, M. Sabbaghian, H. Yanikomeroglu, and A. Yongaçoglu, "Efficient 3D Aerial Base Station Placement Considering Users Mobility by Reinforcement Learning," CoRR, vol. abs/1801.07472, 2018. [Online]. Available: http://arxiv.org/abs/1801. 07472

[31] A. Al-Hourani, S. Kandeepan, and S. Lardner, "Optimal LAP Altitude for Maximum Coverage," IEEE Wireless Communications Letters, vol. 3, no. 6, pp. 569-572, Dec 2014

[32] M. Mozaffari, W. Saad, M. Bennis, and M. Debbah, "Unmanned Aerial Vehicle With Underlaid Device-to-Device Communications: Performance and Tradeoffs," IEEE Transactions on Wireless Communications, vol. 15, no. 6, pp. 3949-3963, June 2016.

[33] V. Sharma, M. Bennis, and R. Kumar, "UAV-Assisted Heterogeneous Networks for Capacity Enhancement," IEEE Communications Letters, vol. 20, no. 6, pp. 1207-1210, June 2016 .

[34] V. Sharma, R. Sabatini, and S. Ramasamy, "UAVs Assisted Delay Optimization in Heterogeneous Wireless Networks," IEEE Communications Letters, vol. 20, no. 12, pp. 2526-2529, Dec 2016.

[35] C. Zhang and W. Zhang, "Spectrum Sharing for Drone Networks," IEEE Journal on Selected Areas in Communications, vol. 35, no. 1, pp. 136144, Jan 2017. 
[36] E. Kalantari, H. Yanikomeroglu, and A. Yongacoglu, "On the Number and 3D Placement of Drone Base Stations in Wireless Cellular Networks," in 2016 IEEE 84th Vehicular Technology Conference (VTCFall), Sept 2016, pp. 1-6.

[37] M. Alzenad, A. El-Keyi, F. Lagum, and H. Yanikomeroglu, "3-D Placement of an Unmanned Aerial Vehicle Base Station (UAV-BS) for Energy-Efficient Maximal Coverage," IEEE Wireless Communications Letters, vol. 6, no. 4, pp. 434-437, Aug 2017.

[38] M. Alzenad, A. El-Keyi, and H. Yanikomeroglu, "3-D Placement of an Unmanned Aerial Vehicle Base Station for Maximum Coverage of Users With Different QoS Requirements," IEEE Wireless Communications Letters, vol. 7, no. 1, pp. 38-41, Feb 2018.

[39] M. Mozaffari, W. Saad, M. Bennis, and M. Debbah, "Wireless Communication Using Unmanned Aerial Vehicles (UAVs): Optimal Transport Theory for Hover Time Optimization," IEEE Transactions on Wireless Communications, vol. 16, no. 12, pp. 8052-8066, Dec 2017.

[40] I. Bor-Yaliniz, S. S. Szyszkowicz, and H. Yanikomeroglu, "EnvironmentAware Drone-Base-Station Placements in Modern Metropolitans," IEEE Wireless Communications Letters, vol. 7, no. 3, pp. 372-375, June 2018.

[41] F. Lagum, I. Bor-Yaliniz, and H. Yanikomeroglu, "Strategic Densification With UAV-BSs in Cellular Networks," IEEE Wireless Communications Letters, vol. 7, no. 3, pp. 384-387, June 2018.

[42] R. I. Bor-Yaliniz, A. El-Keyi, and H. Yanikomeroglu, "Efficient 3-d placement of an aerial base station in next generation cellular networks," in 2016 IEEE International Conference on Communications (ICC), May 2016, pp. $1-5$.

[43] E. Kalantari, M. Z. Shakir, H. Yanikomeroglu, and A. Yongacoglu, "Backhaul-Aware Robust 3D Drone Placement in 5G+ Wireless Networks," in 2017 IEEE International Conference on Communications Workshops (ICC Workshops), May 2017, pp. 109-114.

[44] M. Mozaffari, W. Saad, M. Bennis, and M. Debbah, "Efficient Deployment of Multiple Unmanned Aerial Vehicles for Optimal Wireless Coverage," IEEE Communications Letters, vol. 20, no. 8, pp. 16471650, Aug 2016.

[45] X. Zhou, J. Guo, S. Durrani, and H. Yanikomeroglu, "Uplink Coverage Performance of an Underlay Drone Cell for Temporary Events," CoRR, vol. abs/1801.05948, 2018. [Online]. Available: http://arxiv.org/abs/1801.05948

[46] M. Gruber, "Role of Altitude when Exploring Optimal Placement of UAV Access Points," in 2016 IEEE Wireless Communications and Networking Conference, April 2016, pp. 1-5.

[47] C. W. Chou and L. H. Yen, "Autonomous Deployment of UAVs as Access Points to Serve Wireless Terminals," in 2017 IEEE 86th Vehicular Technology Conference (VTC-Fall), Sept 2017, pp. 1-5.

[48] J. Roberts, "Traffic theory and the internet," Communications Magazine, IEEE, vol. 39, no. 1, pp. 94-99, Jan 2001.

[49] H. Klessig, D. Öhmann, A. J. Fehske, and G. P. Fettweis, "A Performance Evaluation Framework for Interference-Coupled Cellular Data Networks," IEEE Transactions on Wireless Communications, vol. 15, no. 2, pp. 938-950, Feb 2016.

[50] R. S. Sutton and A. G. Barto, Reinforcement learning: An introduction. MIT press, 2018.

[51] J. Schulman, S. Levine, P. Abbeel, M. I. Jordan, and P. Moritz, "Trust region policy optimization." in Icml, vol. 37, 2015, pp. 1889-1897.

[52] J. Schulman, F. Wolski, P. Dhariwal, A. Radford, and O. Klimov, "Proximal policy optimization algorithms," arXiv preprint arXiv:1707.06347, 2017.

[53] H. Klessig, V. Suryaprakash, O. Blume, A. Fehske, and G. Fettweis, "A framework enabling spatial analysis of mobile traffic hot spots," IEEE Wireless Communications Letters, vol. 3, no. 5, pp. 537-540, Oct 2014.

[54] D. Lee, S. Zhou, and Z. Niu, "Spatial modeling of scalable spatiallycorrelated log-normal distributed traffic inhomogeneity and energyefficient network planning," in 2013 IEEE Wireless Communications and Networking Conference (WCNC), April 2013, pp. 1285-1290.
[55] M. Alzenad, A. El-Keyi, F. Lagum, and H. Yanikomeroglu, "3-d placement of an unmanned aerial vehicle base station (uav-bs) for energyefficient maximal coverage," IEEE Wireless Communications Letters, vol. 6, no. 4, pp. 434-437, 2017.

[56] M. Mozaffari, W. Saad, M. Bennis, and M. Debbah, "Efficient deployment of multiple unmanned aerial vehicles for optimal wireless coverage," IEEE Communications Letters, vol. 20, no. 8, pp. 1647-1650, 2016.

[57] "OSM Plugin for Blender". [Online]. Available: https://github.com/ vvoovv/blender-osm

[58] "Blender Project". [Online]. Available: https://www.blender.org/

[59] G. Brockman, V. Cheung, L. Pettersson, J. Schneider, J. Schulman, J. Tang, and W. Zaremba, "Openai gym," arXiv preprint arXiv:1606.01540, 2016.

[60] M. Abadi, P. Barham, J. Chen, Z. Chen, A. Davis, J. Dean, M. Devin, S. Ghemawat, G. Irving, M. Isard et al., "Tensorflow: A system for largescale machine learning," in 12th \{USENIX\} Symposium on Operating Systems Design and Implementation (\{OSDI\} 16), 2016, pp. 265-283.

[61] E. Liang, R. Liaw, R. Nishihara, P. Moritz, R. Fox, J. Gonzalez, K. Goldberg, and I. Stoica, "Ray rllib: A composable and scalable reinforcement learning library," arXiv preprint arXiv:1712.09381, 2017.

[62] J. Schaer, "The densest packing of 9 circles in a square," Canadian Mathematical Bulletin, vol. 8, no. 3, pp. 273-277, 1965. 